\title{
Productivity analysis of horizontal wells intercepted by multiple finite-conductivity fractures
}

\author{
Wang Xiaodong ${ }^{*}$, Li Guanghe ${ }^{2}$ and Wang Fei ${ }^{3}$ \\ ${ }^{1}$ School of Energy Resources, China University of Geosciences, Beijing 100083, China \\ ${ }^{2}$ China University of Petroleum, Beijing 102249, China \\ ${ }^{3}$ Heriot-Watt University, Edinburgh, England
}

(C) China University of Petroleum (Beijing) and Springer-Verlag Berlin Heidelberg 2010

\begin{abstract}
Horizontal wells in the anisotropic reservoirs can be stimulated by hydraulic fracturing in order to create multiple finite-conductivity vertical fractures. Several methods for evaluating the productivity of the horizontal wells have been presented in the literature. With such methods, however, it is still difficult to obtain an accurate result. This paper firstly presents the dimensionless conductivity theory of vertical fractures. Then models for calculating the equivalent wellbore radius and the skin factor due to flow convergence to the well bore are proposed after analyzing the steady-state flow in porous reservoirs. By applying the superposition principle to the pressure drop, a new method for evaluating the productivity of horizontal wells intercepted by multiple finite-conductivity fractures is developed. The influence of fracture conductivity and fracture half length on the horizontal well productivity is quantitatively analyzed with a synthetic case. Optimum fracture number and fracture space are further discussed in this study. The results prove that the method outlined here should be useful to design optimum fracturing of horizontal wells.
\end{abstract}

Key words: Production rate analysis, fractured horizontal wells, finite-conductivity vertical fractures, fracturing design optimization

\section{Introduction}

Compared with vertical wells, horizontal wells are suitable for thin reservoirs, low-permeability reservoirs, and heavy oil reservoirs. However, they fail for anisotropic reservoirs, where the vertical permeability is much less than the horizontal permeability. The advantage of horizontal wells diminishes with an increase in reservoir anisotropy. This situation can be improved by staged fracturing, which generates a number of vertical hydraulic fractures to intersect the horizontal wells, as shown in Fig. 1. Those intersected vertical fractures can reform the flow regimes near the well and increase the vertical permeability of the reservoir, thus enlarging the drainage area of the well. It has been proved that with multiple vertical hydraulic fractures, horizontalwell productivity can be increased significantly (Giger, 1986, 1987; Joshi, 1986; Soliman et al, 1988).

Current methods for productivity evaluation of horizontal wells have their drawbacks (Brown and Economides, 1992; Raghavan and Joshi, 1993; Lang et al, 1994; Raghavan et al, 1997; Xu et al, 2006; Zeng et al, 2007). With such methods, the fracture conductivity is either assumed to be infinite which leads to overestimate the productivity, or treated as

*Corresponding author. email: wxd_cug@cugb.edu.cn Received July 18, 2009 finite in which a complicated Fredholm integral equation must be solved with boundary integral methods. In this paper, the equivalent wellbore radius and the skin due to flow convergence are proposed. By applying the superposition principle, a new analytical method is developed for evaluating the productivity of horizontal wells with multiple finiteconductivity vertical fractures.

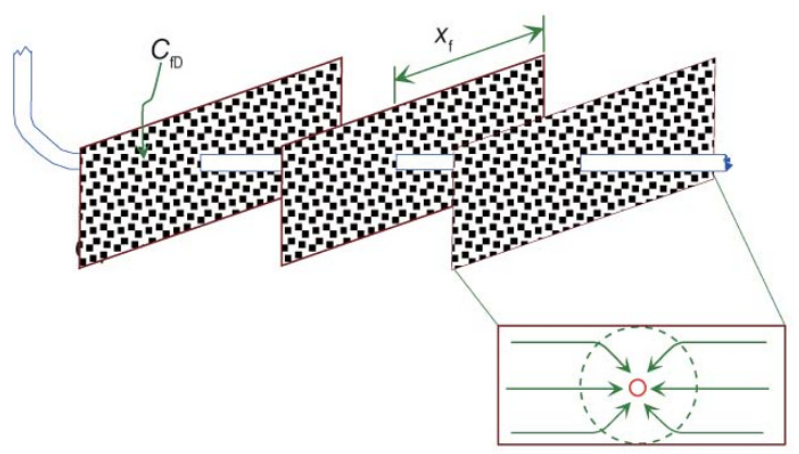

Fig. 1 Schematic of flow regimes in the fracture

\section{Theory of fracture conductivity}

In the process of hydraulic fracturing, sand proppants need to be injected into fractures or the fracture surface acidified in order to maintain the conductive channels after 
the fracturing pressure is removed. The fracture conductivity can be defined quantitatively with the hydraulic fracture conductivity, $C_{\mathrm{f}}$, which is a multiplication product of fracture width $(w)$ and fracture permeability $\left(k_{\mathrm{f}}\right)$, given by Prats et al (1960) as below:

$$
C_{\mathrm{f}}=k_{\mathrm{f}} w
$$

where $C_{\mathrm{f}}$ is the flow rate for unit viscosity fluid passing through a unit-height cross section of the fracture per unit pressure gradient; $k_{\mathrm{f}}$ is the permeability of the propped fracture, $10^{-3} \mathrm{~mm}^{2}$; and $w_{\mathrm{f}}$ is the average fracture width, $\mathrm{m}$.

In reservoir engineering, with respect to hydraulic fracture conductivity, fractures can be divided into three types: infinite-conductivity fractures, uniform-flow fractures, and finite-conductivity fractures. Infinite-conductivity fractures imply that the conductivity is infinite and there is no pressure drop when the fluid flows along the fracture in any instant. Uniform-flow fractures imply that the flow rate along the unit cross section of the fracture is uniform. Finite-conductivity fractures consider that the conductivity is finite and the pressure drops when the fluid flows along the fracture but the flow rate along the unit cross section of the fracture is not uniform. Obviously, the finite-conductivity fracture is a representative type, which is closer to reality.

Theoretical analysis shows that there are two factors which influence productivity enhancement, namely, the dimensionless penetration ratio $\left(1 / r_{\mathrm{eD}}\right)$ and the dimensionless fracture conductivity $\left(C_{\mathrm{fD}}\right)$, which can be expressed as follows:

$$
\begin{aligned}
& r_{\mathrm{eD}}=\frac{r_{\mathrm{e}}}{x_{\mathrm{f}}} \\
& C_{\mathrm{fD}}=\frac{k_{\mathrm{f}} w_{\mathrm{f}}}{k x_{\mathrm{f}}}
\end{aligned}
$$

where $r_{\mathrm{e}}$ is the reservoir drainage radius, $\mathrm{m} ; x_{\mathrm{f}}$ is the fracture half-length, $\mathrm{m}$; and $k$ is the reservoir permeability, $10^{-3} \mu \mathrm{m}^{2}$.

For a given reservoir-fractured well system, the dimensionless fracture conductivity has much physical significance. Assuming that the fracture penetrates the reservoir formation completely so that the fracture height $h_{\mathrm{f}}$ is equal to the formation thickness $h$, the dimensionless conductivity can be reformatted as below:

$$
C_{\mathrm{fD}}=\frac{k_{\mathrm{f}} w_{\mathrm{f}}}{k x_{\mathrm{f}}} \cdot \frac{h_{\mathrm{f}} / \mu}{h / \mu}=\frac{w_{\mathrm{f}} h_{\mathrm{f}}\left(k_{\mathrm{f}} / \mu\right)}{x_{\mathrm{f}} h(k / \mu)}=\frac{q_{\text {inf }}}{q_{\text {outf }}}
$$

where $\mu$ is the fluid viscosity, $\mathrm{mPa} \cdot \mathrm{s}$.

From Darcy's law, $q_{\text {inf }}$ is the inflow rate of fluids into the fracture per unit-pressure gradient, while $q_{\text {out }}$ is the outflow rate of fluids out from the fracture per unit-pressure gradient. Therefore, the fracture conductivity is actually a ratio of flow rate of fluids flowing in and out of the fracture per unit-pressure gradient. If the inflow matches the outflow, the fracture is deemed to be optimum. Considering the importance of fracture conductivity, it must be taken into account when conducting productivity analysis and fracture geometry optimization.

\section{Equivalent wellbore radius}

As we know, the flow performance in horizontal wells with multiple finite-conductivity fractures is complicated. Referring to the approaches of Prats et al (1960) and CincoLey and Samaniego-V (1981), the fluid flow performance in fractures is treated as a combination of linear flow and radial flow, as shown in Fig. 1. After this, based on the general productivity formula of finite-conductivity vertical factures (Wang et al, 2004), a new method for analyzing the productivity of horizontal wells with multiple vertical fractures is developed by introducing equivalent wellbore radius together with skin due to the convergence of fluids into horizontal wellbore.

By solving the integral equation and asymptotic analysis, Wang et al (2004) presented a general productivity formula for wells with finite-conductivity vertical fractures, shown as below:

$$
q_{\mathrm{f}}=\frac{k h\left(P_{\mathrm{avg}}-P_{\mathrm{wf}}\right)}{1.842 \times 10^{-3} \mu B\left[\ln \frac{r_{\mathrm{e}}}{2 x_{\mathrm{f}}}+\frac{3}{4}+f\left(C_{\mathrm{fD}}\right)\right]}
$$

with

$$
f\left(C_{\mathrm{fD}}\right)=\sum_{n=1}^{\infty} \frac{\pi C_{\mathrm{fD}}}{n\left[2 n+\pi C_{\mathrm{fD}}(n+1)\right]}-\frac{\pi C_{\mathrm{fD}}}{\pi C_{\mathrm{fD}}+2}
$$

where $q_{\mathrm{f}}$ is the rate, $\mathrm{m}^{3} / \mathrm{d} ; k$ is the permeability, $\mathrm{mD} ; h$ is the formation thickness, $\mathrm{m} ; B$ is the volume factor, $\mathrm{m}^{3} / \mathrm{m}^{3} ; r_{\mathrm{e}}$ is the drainage radius, $\mathrm{m} ; f\left(C_{\mathrm{fD}}\right)$ is a function of dimensionless conductivity.

The combination of Eq. (5) with the productivity equation for vertical wells producing at pseudo-steady-state gives:

$$
\ln \frac{r_{\mathrm{e}}}{r_{\mathrm{we}}}-\frac{3}{4}=\ln \frac{r_{\mathrm{e}}}{2 x_{\mathrm{f}}}+\frac{3}{4}+f\left(C_{\mathrm{fD}}\right)
$$

Rearranging Eq. (7) yields

$$
r_{\mathrm{we}}=2 x_{\mathrm{f}} \exp \left\{-\left[\frac{3}{2}+f\left(C_{\mathrm{fD}}\right)\right]\right\}
$$

where $r_{\text {we }}$ is the equivalent wellbore radius of a well with finite-conductivity vertical fractures at the pseudo-steady state.

The computational results from Eq. (8) are consistent with the numerical results from the model proposed by CincoLey and Samaniego-V (1981), as shown in Fig. 2. In the productivity analysis, a well with finite-conductivity vertical fractures can be equivalent to a general vertical well by introducing the equivalent wellbore radius model.

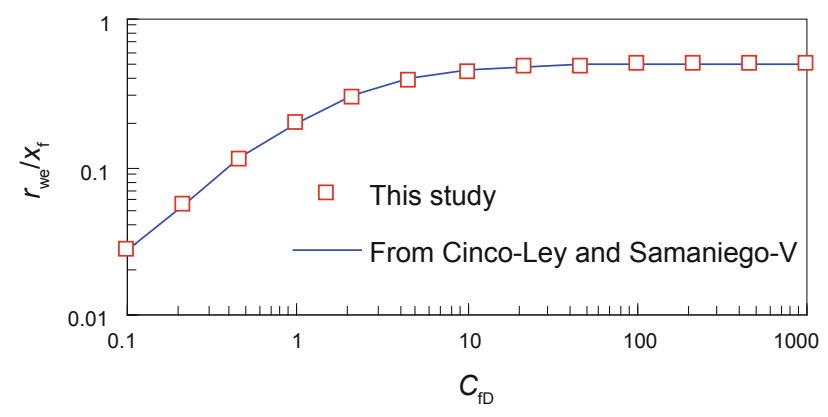

Fig. 2 A comparison of equivalent wellbore radii from different models 


\section{Skin factor due to flow convergence to the wellbore}

As shown in Fig. 1, the fluid flow pattern in hydraulically fractured horizontal wells is special because the fluid flow is comprised of linear flow and radial flow, which is different from that in vertical wells. For vertical wells, the vertical fractures are in lateral contact with the wellbore so there is only linear flow in the fractures. Generally, Eq. (8) is only for a vertical well with vertical fractures. If it is applied to a horizontal well with vertical fractures, the additional pressure drop due to the convergence of fluids into the wellbore should be considered.

The steady-state pressure drop $\left(\Delta P_{\mathrm{R}}\right)$ due to the radial flow in a medium with an outer radius of $h / 2$ (half-reservoir height and a thickness of $w$ (fracture width) can be written as below:

$$
\Delta P_{\mathrm{R}}=\frac{q \mu}{2 \pi k_{\mathrm{f}} w} \ln \frac{h}{2 r_{\mathrm{w}}}
$$

where $r_{\mathrm{w}}$ is the wellbore radius, $\mathrm{m} ; q$ is the rate.

The pressure drop $\Delta P_{\mathrm{L}}$ due to the linear flow in the medium of a width of $h / 2$, thickness of $w$, and length of $h$, can be written as below:

$$
\Delta P_{\mathrm{L}}=\frac{(q / 2) \mu(h / 2)}{k_{\mathrm{f}} w h}
$$

The combination of Eq. (9) with Eq. (10) gives the pressure-drop difference:

$$
\begin{aligned}
\Delta P_{\mathrm{s}} & =\Delta P_{\mathrm{R}}-\Delta P_{\mathrm{L}}=\frac{q \mu}{2 \pi k h}\left[\frac{k h}{k_{\mathrm{f}} w}\left(\ln \frac{h}{2 r_{\mathrm{w}}}-\frac{\pi}{2}\right)\right] \\
& =\frac{q \mu}{2 \pi k h}\left[\frac{1}{C_{\mathrm{fD}}} \frac{h}{x_{\mathrm{f}}}\left(\ln \frac{h}{2 r_{\mathrm{w}}}-\frac{\pi}{2}\right)\right]
\end{aligned}
$$

The skin factor due to the convergence of fluids is defined as (Brown and Economides, 1992):

$$
S_{\mathrm{c}}=\frac{1}{C_{\mathrm{fD}}} \frac{h}{x_{\mathrm{f}}}\left(\ln \frac{h}{2 r_{\mathrm{w}}}-\frac{\pi}{2}\right)
$$

Then the equivalent wellbore radius of horizontal wells with a single vertical fracture can be expressed as:

$$
r_{\mathrm{we}}=2 x_{\mathrm{f}} \exp \left\{-\left[\frac{3}{2}+f\left(C_{\mathrm{fD}}\right)+S_{\mathrm{c}}\right]\right\}
$$

If the damage of fracture surface can be determined, the skin due to the damage can also be introduced into Eq. (12).

\section{Productivity of horizontal wells intercepted by multiple finite-conductivity fractures}

A horizontal well, located at the center of a circular reservoir, is completed with staged fracturing treatments. The drainage radius of the reservoir is $r_{\mathrm{e}}$. The maximum halflength of the fracture is $x_{\mathrm{fmax}}$, and the distance between two neighboring fractures is $d\left(d>x_{\text {fmax }}\right)$. Using the equivalent wellbore radius model and the pressure-drop superposition principle, the steady-state productivity of fractures can be calculated.

\subsection{Horizontal wells with 5 fractures}

A specific case presented by Raghavan and Joshi (1993) was considered, which shows a horizontal well with five infinite-conductivity fractures. As shown in Fig. 3, Fractures 1 and 5 have the longest half length $x_{\mathrm{fl}}$, Fractures 2 and 4 have the second-longest half length $x_{\mathrm{f} 2}$, and Fracture 3 has the shortest half length $x_{\mathrm{f} 3}$. We further assume that $q_{1}$ is the production rate for Fractures 1 and $5, q_{2}$ is the production rate for Fractures 2 and 4 , and $q_{3}$ is the production rate for Fracture 3. If $p_{\mathrm{w}}$ is the bottom-hole flowing pressure, by applying the pressure-drop superposition to this specific system, we have

$$
\begin{aligned}
& p_{\mathrm{w}}-C=\frac{\mu B}{542.87 k h}\left(q_{1} \ln 4 d r_{\mathrm{we} 1}+q_{2} \ln 3 d^{2}+q_{3} \ln 2 d\right) \\
& p_{\mathrm{w}}-C=\frac{\mu B}{542.87 k h}\left(q_{1} \ln 3 d^{2}+q_{2} \ln 2 d r_{\mathrm{we} 2}+q_{3} \ln d\right) \\
& p_{\mathrm{w}}-C=\frac{\mu B}{542.87 k h}\left(q_{1} \ln 4 d^{2}+q_{2} \ln d^{2}+q_{3} \ln r_{\mathrm{we} 3}\right) \\
& p_{\mathrm{e}}-C=\frac{q_{\mathrm{t}} \mu B}{542.87 k h} \ln r_{\mathrm{e}}
\end{aligned}
$$

where $r_{\text {wej }}(j=1,2,3)$ is the equivalent wellbore radius of Fracture $j$ and its appropriate value can be obtained from Eq. (12). The total production rate is given by Raghavan and Joshi (1993):

$$
q_{\mathrm{t}}=\frac{542.87 k h\left(p_{\mathrm{e}}-p_{\mathrm{w}}\right)}{\mu B} /\left[\ln \frac{r_{\mathrm{e}}}{\left(4 d r_{\mathrm{we} 1}\right)^{q_{r 1}}\left(3 d^{2}\right)^{q_{r 2}}(2 d)^{q_{r 3}}}\right]
$$

where $q_{r j}=q_{j} / q_{\mathrm{t}}$.

The ratios $q_{r 1}: q_{r 2}: q_{r 3}$ is expressed as follows:

$$
\begin{aligned}
q_{r 1}: q_{r 2}: q_{r 3} & =\left(\ln 2 \ln 3-\ln \frac{2 r_{\mathrm{we} 2}}{3 d} \ln \frac{r_{\mathrm{we} 3}}{2 d}\right) \\
& :\left(\ln \frac{r_{\mathrm{we} 3}}{2 d} \ln \frac{3 d}{4 r_{\mathrm{we} 1}}+\ln \frac{d}{r_{\mathrm{we} 1}} \ln 2\right) \\
: & \left(\ln \frac{d}{r_{\mathrm{we} 1}} \ln \frac{2 r_{\mathrm{we} 2}}{3 d}+\ln 3 \ln \frac{3 d}{4 r_{\mathrm{we} 1}}\right)
\end{aligned}
$$

The equations above can be easily calculated. Roberts et al (1991) recommend that the minimum distance, $d$, between fractures be greater than $2 x_{\mathrm{f}}$. Then the correlations between

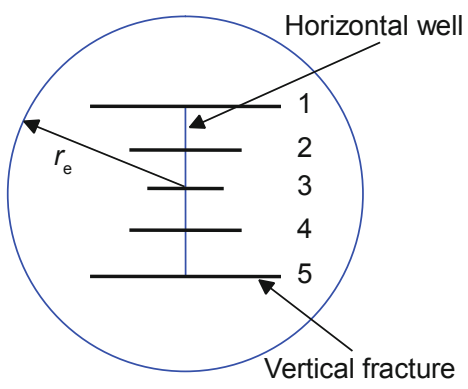

Fig. 3 Schematic of the fractured horizontal well (after Raghavan and Joshi, 1993) 
total flow rate and fractional flow rate can be obtained from Eq. (18). Similarly, the method we used above can be easily applied to other complicated situations.

\subsection{Horizontal well with $N$ fractures}

We use $q_{i}$ to denote the production rate of Fracture $i, d_{j i}$ to denote the distance between Fracture $j$ and Fracture $i$, and $r_{\text {we } i}$ to denote the equivalent wellbore radius of Fracture $i$. By applying the pressure-drop superposition to the well with $N$ fractures, we have

$$
\left\{\begin{aligned}
p_{\mathrm{w}}-C= & \frac{\mu B}{542.87 k h} \times \\
& \left(q_{1} \ln r_{\mathrm{we} 1}+q_{2} \ln d_{21}+q_{3} \ln d_{31}+\cdots+q_{N} \ln d_{N 1}\right) \\
p_{\mathrm{w}}-C= & \frac{\mu B}{542.87 k h} \times \\
& \left(q_{1} \ln d_{12}+q_{2} \ln r_{\mathrm{we} 2}+q_{3} \ln d_{32}+\cdots+q_{N} \ln d_{N 2}\right) \\
p_{\mathrm{w}}-C= & \frac{\mu B}{542.87 k h} \times \\
& \left(q_{1} \ln d_{13}+q_{2} \ln d_{23}+q_{3} \ln r_{\mathrm{we} 3}+\cdots+q_{N} \ln d_{N 3}\right) \\
\vdots & \frac{\mu B}{542.87 k h} \times \\
p_{\mathrm{e}}-C= & \left(q_{1} \ln r_{\mathrm{e}}+q_{2} \ln r_{\mathrm{e}}+q_{3} \ln r_{\mathrm{e}}+\cdots+q_{N} \ln r_{\mathrm{e}}\right)
\end{aligned}\right.
$$

The total production rate $q_{\mathrm{t}}$ of the well is:

$$
q_{\mathrm{t}}=q_{1}+q_{2}+\cdots+q_{N}
$$

By introducing the dimensionless productivity index $J_{i}$, equations above are rearranged as follows:

$$
\left\{\begin{aligned}
0 & =J_{1} \ln \frac{r_{\mathrm{we} 1}}{d_{12}}+J_{2} \ln \frac{d_{21}}{r_{\mathrm{we} 2}}+J_{3} \ln \frac{d_{31}}{d_{32}}+\cdots+J_{N} \ln \frac{d_{N 1}}{d_{N 2}} \\
0 & =J_{1} \ln \frac{r_{\mathrm{we} 1}}{d_{13}}+J_{2} \ln \frac{d_{21}}{d_{23}}+J_{3} \ln \frac{d_{31}}{r_{\mathrm{we} 3}}+\cdots+J_{N} \ln \frac{d_{N 1}}{d_{N 3}} \\
0 & =J_{1} \ln \frac{r_{\mathrm{we} 1}}{d_{14}}+J_{2} \ln \frac{d_{21}}{d_{24}}+J_{3} \ln \frac{d_{31}}{d_{34}}+\cdots+J_{N} \ln \frac{d_{N 1}}{d_{N 4}} \\
\vdots & \\
1 & =J_{1} \ln \frac{r_{\mathrm{e}}}{r_{\mathrm{we} 1}}+J_{2} \ln \frac{r_{\mathrm{e}}}{d_{21}}+J_{3} \ln \frac{r_{\mathrm{e}}}{d_{31}}+\cdots+J_{N} \ln \frac{r_{\mathrm{e}}}{d_{N 1}}
\end{aligned}\right.
$$

where the dimensionless productivity index is defined as

$$
J_{i}=\frac{q_{i} \mu B}{542.87 k h\left(p_{\mathrm{e}}-p_{\mathrm{w}}\right)} \quad i=1,2, \cdots, N
$$

The dimensionless productivity index $J_{i}$ of each fracture is obtained by solving equations above, and then the production rate of each fracture is determined.

\subsection{Case study}

For this case, a horizontal well is stimulated by four vertical fractures. The well is drilled at the center of a homogeneous reservoir. Firstly, the influence of the half length and dimensionless conductivity on the well productivity is presented. Then, the influence of the fracture number on the total well production-rate and each fracture production-rate is tested.

Essential parameters for this case are available: $\mu=20.0$ $\mathrm{mPa} \cdot \mathrm{s}, r_{\mathrm{w}}=0.1 \mathrm{~m}, B=1.1, k=10 \mathrm{mD}, 2 L=400.0 \mathrm{~m}, h=10.0$ $\mathrm{m}, z_{\mathrm{w}}=5.0 \mathrm{~m}, r_{\mathrm{e}}=350.0 \mathrm{~m}\left(z_{\mathrm{w}}\right.$ is the well location in the $z$ direction, $L$ is the horizontal-well half length). By solving Eq. (22) presented above, the results for this case are obtained, as shown in Fig. 4 through Fig. 6.

In this case, of which four fractures have identical length and uniform distribution, shown in Fig. 4 and Fig. 5, the outermost fractures have higher productivity indexes due to the larger drainage area. The inner fractures have lower productivity indexes due to the interference from outer fractures. Notice that the larger the available drainage area, the more sufficient the fluid flow from the formation into the fracture, and the greater the well production will be. The influence of the half length and dimensionless conductivity of the fracture on outer fracture productivity is bigger than that on inner ones. So in order to achieve a uniform production profile, one can first adjust the facture length, and then adjust the dimensionless fracture conductivity and fracture space.

As shown in Fig. 6, with the increase in the fracture number, the well production rate increases rapidly at the beginning and almost stabilizes later. The output of each fracture decreases as the fracture number increases. Based on

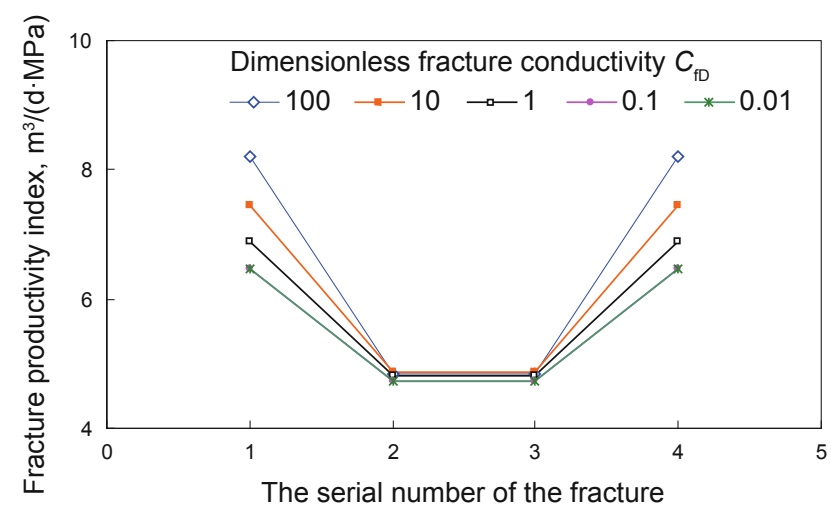

Fig. 4 Curves of productivity index varying with $C_{\mathrm{fD}}$

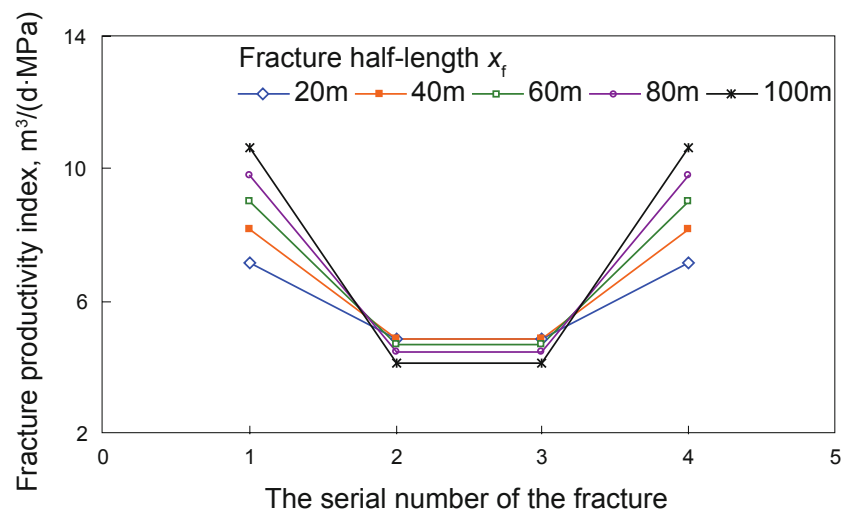

Fig. 5 Curves of productivity index varying with $x_{\mathrm{f}}$ values 
this result, the optimum fracture number can be obtained with the combination of production-rate trends with the economic conditions. In this case, the optimum fracture number is five, as marked in Fig. 6.

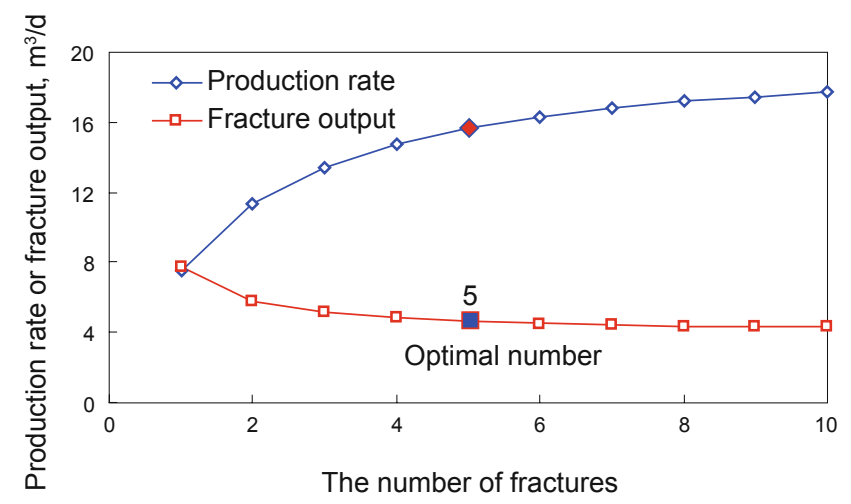

Fig. 6 Production rate and fracture output varying with fracture number

In addition, we notice that the effect of flow convergence can be negligible unless the dimensionless fracture conductivity is very small or the half length is very short.

\section{Conclusions}

1) Dimensionless fracture conductivity is the key parameter which affects the fracture efficiency. It is a ratio of the inflow to the outflow of the fluid in the fracture under unit-pressure gradient. Based on this value, the fracture geometry can be optimally designed.

2) An equivalent wellbore radius is obtained by combining productivity formulas for fractured wells and normal vertical wells in accordance with the production-equivalent principle. The accuracy of this model is proved and the significant influence of dimensionless fracture conductivity on well productivity is further verified.

3) The typical flow pattern of horizontal wells within a fracture is radial flow followed by linear flow. Based on this theory, the skin due to flow convergence is introduced and thus a new method for evaluating the productivity of horizontal wells with multiple finite-conductivity vertical fractures is developed. It has been proved that the skin due to flow convergence normally has a negligible effect on the well productivity.

4) It has been proved that there is mutual interference among the fractures. For the fractures with identical length and proportional space, the outermost fractures contribute more than the middle ones to enhance the production. The total production rate of the well increases as the fracture number increases, but the production rate of each fracture decreases. Optimization design can be operated by combining production rate trends and economic costs.

\section{References}

Brown J E and Economides M J. An analysis of hydraulically fractured horizontal wells. Paper SPE 24322 presented at SPE Rocky Mountain Regional Meeting, 18-21 May 1992, Casper, Wyoming

Cinco-Ley H and Samaniego-V F. Transient pressure analysis for fractured wells. Journal of Petroleum Technology. 1981. 33(9): 17491766 (Paper SPE 7490-PA)

Giger F M. Analytic 2D models of water cresting before breakthrough for horizontal wells. Paper SPE 15378 presented at the 61st Annual Technical Conference and Exhibition of the Society of Petroleum Engineers, 5-8 October 1986, New Orleans, LA

Giger F M. Low-permeability reservoirs development using horizontal wells. Paper SPE 16406 presented at the SPE/DOE Low Permeability Reservoirs Symposium, 18-19 May 1987, Denver, Colorado

Joshi S D. Augmentation of well productivity with slant and horizontal wells. Paper SPE 15375 presented at the 61st Annual Technical Conference and Exhibition of the Society of Petroleum Engineers, 5-8 October 1986, New Orleans, LA

Lang Z X, Zhang L H and Cheng L S. A study of the productivity of fractured horizontal wells. Journal of the University of Petroleum, China. 1994. 18(2): 43-45 (in Chinese)

Prats M, Hazebroek P and Strickler W R. Effect of vertical fractures on reservoir behavior - Incompressible fluid case. Paper SPE 98-PA presented at 35th Annual Fall Meeting of SPE, Oct. 1960, in Denver, USA; also presented at SPE Journal. 1962. 2(2): 87-94

Raghavan R and Joshi S D. Productivity of multiple drainholes or fractured horizontal wells. SPE Formation Evaluation. 1993. 8(1): 11-16 (Paper SPE 21263-PA)

Raghavan R, Chen C C and Agarwal B. An analysis of horizontal wells intercepted by multiple fractures. SPE Journal. 1997. 2(3): 235-245 (Paper SPE 27652)

Roberts B E, van Engen H and van Kruysdijk J W. Productivity of multiple fractured horizontal wells in tight gas reservoirs. Paper SPE 23113 presented at the Offshore Europe Conference, 3-6 September 1991, Aberdeen, United Kingdom

Soliman M Y, Hunt J E and Rabaa A M. Fracturing aspects of horizontal wells in naturally fractured reservoir. Paper SPE 18302 presented at the 63rd Annual Technical Conference and Exhibition of the Society of Petroleum Engineers, 2-5 October 1988, Huston, TX

Wang X D, Zhang Y T and Liu C Q. Productivity evaluation and conductivity optimization for vertically fractured wells. Petroleum Exploration and Development. 2004. 31(6): 78-81 (in Chinese)

Xu Y B, Qi T and Yang F B. New model for productivity test of horizontal wells after hydraulic fracturing. Acta Petrolei Sinica. 2006. 27(1): 89-91 (in Chinese)

Zeng F H, Guo J C and Xu Y B. Factors affecting production capacity of fractured horizontal wells. Petroleum Exploration and Development. 2007. 34(4): 474-477 (in Chinese)

(Edited by Sun Yanhua) 